\title{
Special Session 7 Young Stars, Brown Dwarfs, and Protoplanetary Disks
}

\author{
Jane Gregorio-Hetem ${ }^{1}$ \\ and Silvia Alencar ${ }^{2}$ \\ ${ }^{1}$ Universidade de São Paulo, IAG/USP, Brazil \\ email: jane@astro.iag.usp.br \\ ${ }^{2}$ Departamento de Física, ICEx/UFMG, Brazil, \\ email: silvia@fisica.ufmg.br
}

\section{Preface}

In recent years our knowledge of star, brown dwarf and planet formation has progressed immensely due to new data in the IR domain (Spitzer telescope), new X-ray campaigns such as the Chandra Orion Ultradeep Project (COUP) and the X-ray Emission Survey of Taurus (XEST), with XMM-Newton, as well as adaptive optics results and synoptic studies of young stellar and substellar objects.

Disk accretion and jet outflows, which are intimately associated with the formation and early evolution of stars, has also provided new insights in the early stellar years. High angular resolution observations provide amazing images of protoplanetary disks around young stars at various evolutionary stages and different environments. Jet structures have also been resolved on scales of a few to a hundred AU. More spectacular results related to disk and jet structure and dynamics are already starting to appear from interferometry (VLTI, Keck/I) and improved imaging systems such as HST/ACS.

At the same time, observing programs to find planets by transit, such as the CoRoT mission, are expected to probe a new planetary mass domain and provide new results on planet formation. CoRoT Additional Programmes are also exciting opportunities to study photometric and spectroscopic variability of PMS stars that present a rich variety of types, which includes pulsation, hot and cold spots, higher modes of vibrations, stellar multiplicity, accretion processes, jets, disk debris passing in front of the star, Algol-type occultations, among many others.

Following approval by the IAU Executive Commitee, this IAU Special Session on Young Stars, Brown Dwarfs, and Protoplanetary Disks allowed a meeting with a broad range of subjects linked to star/planet formation and evolution, taking advantage that researchers from different, but closely related areas, attended the IAU General Assembly in 2009. The integration of talks and discussions provided excellent scientific exchange between researchers from different backgrounds to achieve significant debate about open issues in the formation and early evolution of stars, brown dwarfs, and planets.

The papers presented here, in the format of extended abstracts, give a summary of the range of topics covered in our meeting. Most of the authors have consented to make available the slides of their oral presentations at our website, where the poster contributions can also be found: http://www.fisica.ufmg.br/ss7iau09/.

The Scientific Programme benefited from the contribution of the Scientific Organising Committee (S.O.C.), suggesting names of invited speakers that fulfilled the aims of our meeting. The final choice of talk contributions was a hard task for the S.O.C., due to the high demand (77 submitted abstracts for oral contributions) to be fitted in a short 
schedule (2.5 days meeting). In total, the Scientific Programme counted on 9 invited talks, 25 oral presentations, and 79 poster presentations. We estimated about a hundred people attending the Special Session 7 (SpS7).

Unfortunately two of the invited speakers, Ray Jayawardhana and Isabelle Baraffe, could not come. Folowing their suggestions, their talks were presented respectively by Ilaria Pascucci and Subu Mohanty. We are greatful to them, for accepting the substitution role and for doing excellent presentations. A special thank goes to the session chairs Celso Batalha, Suzana Lizano, Hans Zinnecker, Eric Feigelson, Michael Sterzik, and Sylvie Cabrit.

The Local Organising Committee also contributed to the success of the SpS7. We greatly acknowledge Daniela Lazzaro and all the colleagues from Rio de Janeiro, who direct or indirectly helped us in the running of this special session. We also thank Beatriz Barbuy who provided financial support (CNPq Proc. No. 451483/2009) for travel expenses of some participants of the SpS7.

\title{
Scientific Organising Committee
}

\author{
Silvia Alencar (Brazil, Co-chair), \\ Nuria Calvet (USA), \\ Gilles Chabrier (France), \\ Francesca D'Antona (Italy), \\ Eric Feigelson (USA), \\ Jane Gregorio-Hetem (Brazil, Co-chair), \\ Sergei Lamzin (Russia), \\ Susana Lizano (Mexico), \\ Robert Mathieu (USA), \\ Thierry Montmerle (France), \\ Antonella Natta (Italy), \\ Bo Reipurth (USA), \\ Hsien Shang (Taiwan), \\ Michael Sterzik (Chile), \\ Ewine van Dishoeck (Netherlands), \\ Hans Zinnecker (Germany).
}

